\title{
Cloud Storage and Online Bin Packing
}

Doina Bein, Wolfgang Bein, and Swathi Venigella

\begin{abstract}
We study the problem of allocating memory of servers in a data center based on online requests for storage. Given an online sequence of storage requests and a cost associated with serving the request by allocating space on a certain server one seeks to select the minimum number of servers as to minimize total cost. We use two different algorithms and propose a third algorithm. We show that our proposed algorithm performs better for large number of random requests in terms of the variance in the average number of servers.
\end{abstract}

\section{Introduction}

Cloud storage is the service provided by numerous entities to store and backup computer files. We study the cost of storing vast amounts of data on the servers in a data center and we propose a cost measurement together with an algorithm that minimizes such cost. The bin packing optimization problem seeks to minimizes the number of bins a given sequence of items needs to be packed. This problem can apply to storing data on hard-drives in a data centers as follows: In a data center, files need to be stored on hard-drives. Without loss of generality we assume that all the hard-drives have the same size (of 1 unit), since buying them in bulk, of the same size is much cheaper (and easier to be replaced) than buying them individually, with various sizes. In this case, the hard drive represents a bin that needs to receive items to be stored. The goal is to minimize the number of bins (or disks)

Doina Bein

The Pennsylvania State University, Applied Research Laboratory, e-mail: siona@psu.edu

Wolfgang Bein

The University of Nevada, Las Vegas, School of Computer Science, e-mail: beinw@unlv.nevada.edu

Swathi Venigella

The University of Nevada, Las Vegas, School of Computer Science, e-mail: swathiv99@ gmail.com 
used for storing the files. We note that many important applications in areas such as multi-processor scheduling, resource allocation, packet routing, paged computer memory systems, storage, multiprocessor scheduling, stock cutting, loading trucks with certain weight capacities, creating file backup in removable media and technology mapping in FPGA implementation of custom hardware can be modeled as a bin packing optimization problem $[1,2,3,4]$.

We study various bin packing schemes which could be relevant for cloud computing as they can be used for minimizing the energy consolidation [5, 6] in data centers. In Section 2 we present basic notions related to the bin packing problem, give a number of approximation algorithms which solve this problem, and we propose a novel algorithm " $k$-Binary". Simulation results are presented in Section 3. Concluding remarks are in Section 4.

\section{Online Bin Packing}

The bin packing problem requires packing a set of objects into a finite number of bins of capacity $V$ in a way that minimizes the number of bins used: Given a sequence of $n$ items $L=\{1,2, n\}$ with sizes $s_{1}, s_{2}, \ldots, s_{n} \in(0,1]$, one needs to find a partition of the items into sets of size 1 (called bins) so that the number of sets in the partition is minimized [7] and the sum of the sizes of the pieces assigned to any bin may not exceed its capacity. We say that an item that belongs to a given bin (set) is packed into this bin. A bin is empty if no item is packed into it, otherwise it is used. Since the goal is to minimize the number of bins used, we would like to find an algorithm which finds a number of bins that is a constant factor of the minimum possible number of bins, no matter what the input is. Bin packing is NP-hard, thus finding an exact solution for any given input can be done currently only in exponential time.

For a given input sequence $L$ of $n$ items, let $A(L)$ (or simply $A$ ) be the number of bins used by algorithm $A$ on the input sequence $L$. Let $O P T(L)$ (or simply $O P T$ ) be the number of bins used by an optimal offline algorithm which knows the sequence $L$ in advance. The asymptotic performance ratio for an algorithm $A$ is

$$
\lim _{\sup } \rightarrow \infty \sup _{L}\left\{\frac{A(L)}{O P T(L)} \mid O P T(L)=n\right\}
$$

For the general bin packing algorithm we assume that the entire list and its item sizes are known before the packing begins. A common situation is where the items arrive in some order and must be assigned to some bin as soon as they arrive, without knowledge of the remaining items. A bin packing algorithm that can construct its packing under this online regime is called an online bin packing algorithm, for which items in the list must be processed in exactly the same order as they are given, one at a time. The online processing is difficult owing to the fact that unpredictable item sizes may appear. In general, the performance of an online bin packing algorithm is substantially affected by the permutation of items in a given list. 
We present next the three most common and simplest online bin packing algorithms: Next Fit, First Fit, and Best Fit. The asymptotic performance ratio of these algorithms is $r_{\text {NextFit }}=2$, while the $r_{\text {FirstFit }}$ and $r_{\text {FirstFit }}$ are both 1.7 [8].

Next Fit is a bounded space online algorithm in which the only partially filled bin that is open is the most recent one to be started. It uses one active bin into which it packs the input. Once the free space in this bin becomes too small to accommodate the next item, a new active bin is opened and the previous active bin is never used again. This process continues until there are no more elements. First Fit keeps all non-empty bins active and tries to pack every item in these bins before opening a new one. If no bin is found, it opens a new bin and puts the item in the new bin. Best Fit picks the bin with the least amount of free space in which it can still hold the current element. All unfilled bins are kept open until the end. Let $S A(n)$ denote the maximum number of storage locations (for active bins) needed by some algorithm A during the processing of the list $\mathrm{L}$ whose size is $n$, and refer to algorithm $\mathrm{A}$ as an $S A(n)$-space algorithm. Next Fit is an $O(1)$-space algorithm, since it involves only one active bin at all times; both First Fit and Best Fit are an $O(n)$-space algorithms, since they keep all non-empty bins active until the end of the processing [9].

There are other online algorithms, based on a non-uniform partitioning of interval space $(0,1]$ into $M$ sub-intervals, assuming that the size of all items is no larger than 1. We are given a list of items $L=\left\{a_{1}, a_{2}, \ldots, a_{n}\right\}$, each with item size $s\left(a_{i}\right), 0<$ $s\left(a_{i}\right) \leq 1$. The interval $(0,1]$ that represents the content of a bin is partitioned into harmonic sub-intervals $I^{M}=\left\{\left(0, \frac{1}{M}\right],\left(\frac{1}{M}, \frac{1}{M-1}\right], \ldots(1 / 2,1]\right\}$ where $M$ is a positive integer. Then we classify each item according to the size, i.e., if the item size is in the interval $I_{k}=\left(\frac{1}{k-1}, \frac{1}{k}\right], k>1$, then the item is called an $I_{k}$-item; if the item size is in the interval $I_{M}=\left(0, \frac{1}{M}\right]$ then the item is called $I_{M}$-item. We note than in one bin, no more than $k I_{k}$-items can be fit, $k<M$, and each $I_{k}$ filled bin packs exactly $k$ items, irrespective of the actual sizes of these items, $k<M$. Into an $I_{M}$ bin at least $M$ items can fit. We present two most known such algorithms: Harmonic [7] and Cardinality Constrained Harmonic [10]. Later in this section we present our proposed algorithm - also based on a different type of partitioning.

Algorithm Harmonic keeps active an unfilled bin of each type: one bin of $I_{1}$ type items, one bin of $I_{2}$ type items, etc., thus a total of $\mathrm{M}$ bins are active at each moment. When an item arrives, it is packed in the corresponding bin; if the bin is filled, then it is closed and another bin is open, that will store items of that type. Once all the items are packed, we add the number of bins closed and the number of bins unfilled. Harmonic is completely independent of the arriving order of items [7]. A disadvantage of Harmonic is that items of size larger than $1 / 2$ are packed one per bin, possibly wasting a lot of space in each single bin. Harmonic runs in $O(n \log M)$ time and uses $M$ storage spaces for the active bins. Lee and Lee [7] had shown that the worst-case performance ratio of the algorithm is not related to the partition number $M$. Therefore, $M$ can be regarded as a constant, and hence Harmonic is $O(1)$-space and $O(n)$-time algorithm.

Algorithm Cardinality Constrained Harmonic imposes a limit of $M$ on the number of items that can fit into $I_{M}$ bins, irrespective of whether more items can still fit into that bin. Whenever $M$ items are stored in an $I_{M}$-bin, we close the bin and open a 
new one. We note again that the exact size does not affect the packing process, since each bin will contain only items of a single type, irrespective of the exact size.

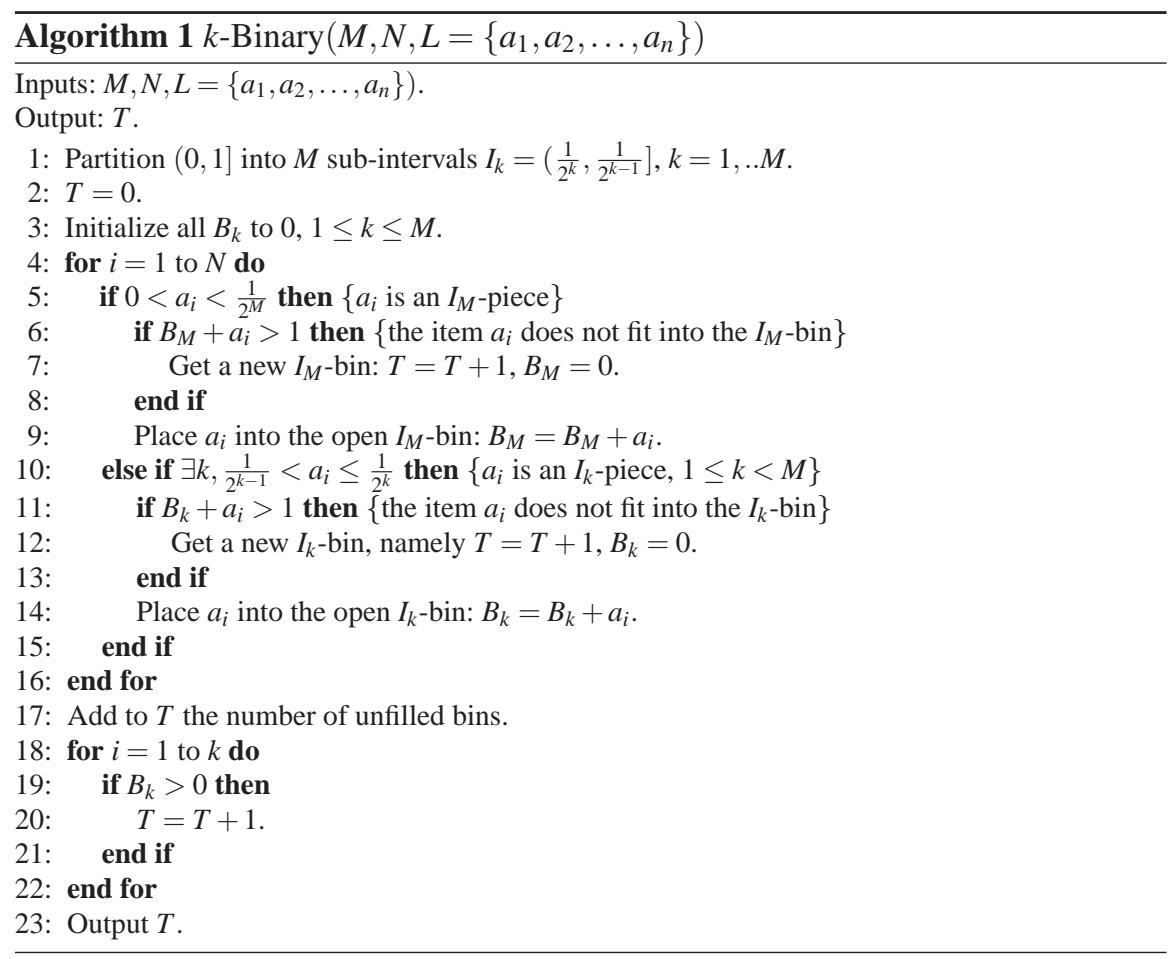

Algorithm $k$-Binary (see algorithm box 1$)$ partitions the interval $(0,1]$ into subintervals in which the endpoints are a negative power of 2: $(0,1]=\cup_{k=1}^{M} I_{k}$ where $I_{k}=\left(\frac{1}{2^{k}}, \frac{1}{2^{k-1}}\right]$ and $k$, the number of partitions, is a positive integer. For example, if $k=3$ then the interval $(0,1]$ is partitioned into three intervals $(0,1 / 4],(1 / 4,1 / 2]$, and $(1 / 2,1]$.

\section{Simulation Results}

Different sequences of requests produce different results when $M$, the number of partitions, varies. We compare the performance of the $k$-binary algorithm with the performance of Harmonic and Cardinality Constrained Harmonic. We measure the efficiency of the implemented algorithms using the average number of bins of any type used. For a given input, let $N$ be the total number of items and $M$ be the total number of partitions. Let us consider $T B^{1}$ to be the total number of bins used by Harmonic, $T B^{2}$ to be the total number of bins used by Cardinality Constrained Har- 
monic and $T B^{3}$ be the total number of bins used by $k$-Binary. For each algorithm we will compute how well the algorithm balances the bins of any type. For an algorithm $j, 1 \leq j \leq 3$, let $N B_{k}^{j}$ be the number of bins of $I_{k}$-type. If the algorithm $j, 1 \leq j \leq 3$, balances the items well, then all $N B_{k}^{j}$ s should be roughly $\frac{T B^{j}}{M}$.

To compare how well an algorithm balances the types of bins used, we compute the variance $V A R$ :

$$
V A R=\sum_{k=1} M\left(N B_{k}^{j}-T B^{j} / M\right)^{2}
$$

We generate random sequence of requests as follows. We first generate a subsequence of $N$ random values in the interval $(0.01,0.99), L_{1}=\left\{a_{1}, a_{2}, \ldots, a_{N}\right\}$, $0.01 \leq a_{i} \leq 0.99$ and we call this the random sub-sequence. We append to $L_{1}$ another sub-sequence of $N$ values, $L 2=\left\{b_{1}, b_{2}, \ldots, b_{N}\right\}$, where $b_{i}=1-a_{i}$, and we call this as the computed sub-sequence. The random sequence contains the random sub-sequence followed by the computed sub-sequence.

We run extensive number of simulations by generating such random sequences of length $2 N$ for various values of $N$ and $M$. We observed that in some cases our proposed Algorithm $k$-Binary requires less number of bins than Harmonic, and there are other cases where $k$-Binary requires more bins than Harmonic. In almost all the cases, Cardinality Constrained Harmonic required at least the same number of bins as Harmonic, if not more. No trend has been observed as for values of $N$ and $M$ which algorithm, $k$-Binary or Harmonic, has the best performance, namely it gives the least number of bins.

We also compared the three algorithms based on the variance in the number of bins used, namely the value of VAR (Equation 1), for small or large sequences of random requests. When the number of items is relatively small, $N<100$, the values for variance are plotted in Figure 1(a). When the number of items is relatively large, $10<N<1000$, the values for variance are plotted in Figure 1(b). In both figures, the $\mathrm{x}$ axis shows the number of bins and the $\mathrm{y}$ axis show the variance value obtained by each algorithm.

We note that for smaller number of items, there is not much variation between the three algorithms. The proposed Algorithm $k$-Binary is as efficient as Harmonic and Cardinality Constrained Harmonic. For larger number of items, we note that $k$-Binary has a lower variance than Harmonic but slightly higher variance than Cardinality Constrained. We can then conclude that the proposed $k$-binary algorithms behaves in average better than both previously proposed algorithms in terms of the variance on the type of bins used.

\section{Conclusion and Future Work}

The algorithm proposed by Lee and Lee has a competitive ratio which is very close to the optimum, but has a high variation on the types of bins used. The algorithm proposed by Epstein has a worse competitive ratio, i.e. uses more bins for packing the items than Lee and Lee, but it has a much smaller variation than Lee and Lee. 


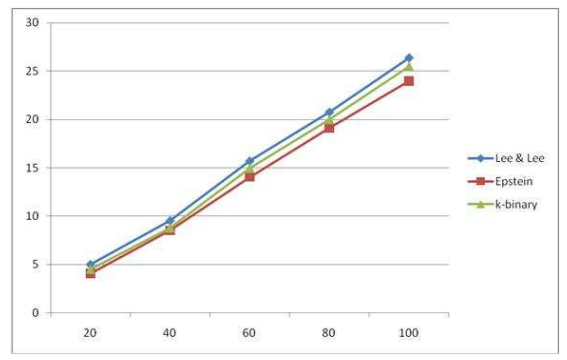

(a) Smaller number of items

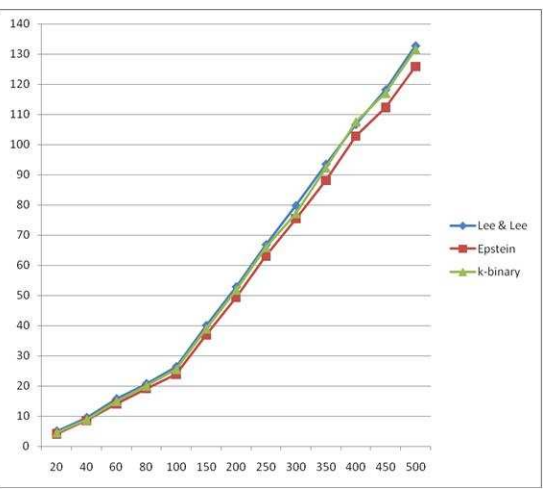

(b) Larger number of items

Fig. 1 Trend of the variance

We propose an algorithm, $k$-Binary, that uses fewer bins than the algorithm proposed by Epstein, and a slightly more bins than the algorithm proposed by Lee and Lee. At the same time, $k$-Binary works better than both previously proposed algorithms. Modern computers have multiple processors, thus it might be of interest to provide algorithms that allow serving two or more requests at a time.

\section{References}

1. J.D. Ullman. The performance of a memory allocation algorithm. Tech. Report 100, Princeton University, Princeton, NJ, 1971.

2. E.G. Coffman Jr., M.R. Garey, and D.S. Johnson. Approximation algorithms for bin packing: An updated survey, pages 46-93. PWS Publishing Co., Boston, MA, USA, 1997.

3. E.G. Coffman Jr. and J. Csirik. Performance guarantees for one-dimensional bin packing, chapter 32. Chapman \& Hall/CRC, 2007.

4. J. Csirik and G.J. Woeginger. On-line packing and covering problems, A. Fiat and G.J. Woeginger (editors), chapter 7, page 147177. Springer, 1998.

5. B. Li, J. Li, J. Huai, T. Wo, Q. Li, and L. Zhong. Enacloud: An energy-saving application live placement approach for cloud computing environments. In IEEE International Conference on Cloud Computing (CLOUD), pages 17-24, 2009.

6. S. Srikantaiah, A. Kansal, and F. Zhao. Energy aware consolidation for cloud computing. In Conference on Power Aware Computing and Systems (HotPower), page 10, 2008.

7. C. Lee and D. Lee. A simple on-line bin-packing algorithm. Journal of ACM, 32:562572, 1985.

8. S.S. Seiden. An optimal online algorithm for bounded space variable-sized bin packing. SIAM Journal on Discrete Mathematics, 14:2001, 2000.

9. M. Drake. Bin packing, 2006.

10. S.S. Seiden, R. Van Stee, and L. Epstein. New bounds for variable-sized online bin-packing. SIAM Journal on Computing, pages 455-469, 2003. 